\title{
Lifestyle risk factors for pressure ulcers in community-based patients with spinal cord injuries in Japan
}

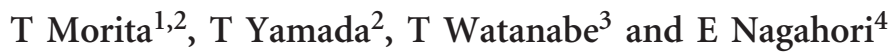

\section{Study design: Case-control study.}

Objectives: To identify daily living-related risk factors for pressure ulcer (PU) occurrence in community-living patients with spinal cord injury (SCI). To determine whether seat pressure influences PU occurrence, and how often patients with $\mathrm{SCl}$ perform pressure relief activities while living in the community.

Setting: Kanagawa Rehabilitation Hospital, Kanagawa, Japan.

Methods: Thirty-one patients admitted to this hospital for PU treatment were included in the case (PU) group. Thirty outpatients who did not have PUs at the time of the study, and had lived without PUs for at least a year, were included in the control (No PU) group. Patients were interviewed about lifestyle-related PU risk factors and a pressure-mapping system was used to measure interface pressure (IP) on their wheelchair seat. The No PU group patients recorded their daily activities and pressure relief maneuvers for 1 week.

Results: Eight lifestyle factors and one risk assessment scale significantly differed between groups. Three factors showed significant odds ratios by logistic regression. IP did not significantly differ between groups. The self-counted number of pressure relief maneuvers (median (25th-75th percentile)) performed per hour in the No PU group was 2.5 (0.7-4.3) and including transfer and urination was 3.5 (2.0-5.3).

Conclusion: We identified potential PU risk factors related to lifestyle. The scores of one risk assessment scale were also associated with PU risk. Our results did not suggest an IP damage threshold. Patients in the No PU group performed pressure relief maneuvers, including related activities, approximately once every $17 \mathrm{~min}$.

Spinal Cord (2015) 53, 476-481; doi:10.1038/sc.2015.18; published online 10 February 2015

\section{INTRODUCTION}

Prevention of secondary complications in patients with spinal cord injury (SCI) is important to allow them to continue living in the community. Pressure ulcers (PUs) are a common secondary complication of SCI in many countries. ${ }^{1-4}$ Although patients with SCI may believe that they can prevent PUs through careful management of living conditions, ${ }^{3}$ in reality PU prevention is very difficult.

Pressure relief and redistribution through correct sitting posture, wheelchair settings and seat cushion selection are important, ${ }^{5,6}$ as is evaluating daily living practices that carry a risk of causing PUs. ${ }^{7}$ In the clinical setting, we contribute to PU prevention by providing pressure redistribution on the wheelchair, instructing patients in pressure relief practices and evaluating activities that include the risk of PUs. ${ }^{8}$ Therefore, knowing which aspects of daily living strongly influence the risk of PU occurrence would help guide prevention strategies. Characterization of the relationship between interface pressure (IP) measurements on the wheelchair and PUs is also essential, ${ }^{9}$ as IP may be useful for the investigating seat pressure and daily living-related risk factors for PUs in patients with SCI.

Pressure relief is important for PU prevention. It is our standard practice to instruct patients with SCI to perform pressure relief, such as a lift or other maneuvers, ${ }^{6}$ every $15 \mathrm{~min}$ throughout the day. ${ }^{10}$ However, it has been difficult to determine how often community-living patients with SCI really perform pressure relief maneuvers once they have finished rehabilitation. Previous reports suggest that community-living SCI patients seldom perform pressure relief. ${ }^{11}$

Thus, the objectives of this study were:

1. To determine which daily living factors strongly influence the risk of PUs among community-living patients with SCI.

2. To determine whether IP influences PU occurrence.

3. To determine how often community-living patients with SCI practice pressure relief.

\section{MATERIALS AND METHODS}

The affected patients (PU group; $n=31$ ) in this case-control study were admitted to the Kanagawa Rehabilitation Hospital for treatment of PUs between January and December of 2011. All were community-living prior to admittance. As their ulcers occurred on the ischial tuberosity, we speculated that their PUs were caused by their seating surface. The patients in the control group (No PU group; $n=30$ ) were outpatients in the same hospital, but did not have PUs at the time of the study and had lived in the community without PUs for at least 1 year. Patients in both groups were matched by sex, level of injury and severity of paralysis as much as possible to allow appropriate comparisons of other factors. Paralysis severity was classified according to the AIS American Spinal Injury Association (ASIA) impairment scale. We interviewed patients

${ }^{1}$ Department of Physical Therapy, Kanagawa Rehabilitation Hospital, Atsugi, Japan; ${ }^{2}$ Department of Physical Therapy, Graduate School of Human Health Sciences, Tokyo Metropolitan University, Arakawa, Japan; ${ }^{3}$ Department of Orthopedics, Kanagawa Rehabilitation Hospital, Atsugi, Japan and ${ }^{4}$ Department of Nursing, Kanagawa Rehabilitation Hospital, Atsugi, Japan

Correspondence: T Morita, Department of Physical Therapy, Kanagawa Rehabilitation Hospital, 516 Nanasawa, Atsugi, Kanagawa 243-0121, Japan.

E-mail: moritatomoyuki170@gmail.com

Received 27 June 2014; revised 19 December 2014; accepted 8 January 2015; published online 10 February 2015 
from both groups and evaluated matching when $>20$ patients per group had been recruited. Subsequently, when selecting patients for the No PU group we matched them for age, ASIA impairment scale and damage level with the PU group patients.

Patient interviews using a structured questionnaire, medical record searches, IP measurements in the wheelchair, and risk assessment using the Braden scale and the SCI pressure ulcer scale (SCIPUS) were conducted for all patients (see Appendix). The reliability of the SCIPUS and Braden scales for the prediction of SCI risk has been demonstrated; however, their validity has not been reported. ${ }^{12}$ The interview questionnaire included questions on sociodemographic status and lifestyle factors, the latter were selected based on previous studies and our clinical experience ${ }^{5-8,10,13}$ and are shown in Table 1. On-thewheelchair IP measurements of both groups were done using the FSA (Force sensing Array) Pressure Mapping System (Vista Medical Ltd, Manitoba, Canada); peak pressure, contact area and average pressure were calculated. Patients in the PU group underwent pressure mapping before surgical treatment if possible, as bone shape may change after surgical PU treatment.

In the No PU group, daily living habits (urination, defecation, bathing, going outside and the length of time spent in a wheelchair) were recorded daily for 1 week. The number of pressure relief maneuvers was also recorded using an electronic tally counter (Line Seiki Co., Ltd., Tokyo, Japan). Push-ups and leaning the trunk forward and to the side were considered pressure relief maneuvers. The length of time spent in pressure relief positions was not measured because it was too difficult to time each position that subjects kept on. Average weekly pressure relief maneuvers were calculated in two ways: 'self-counted' pressure relief was calculated as the number of pressure relief maneuvers per hour (based on the number of counted pressure relief maneuvers and the time spent in the wheelchair); 'including related activities' also included transfer and voiding urine as pressure relief maneuvers in the calculation. These values were compared with the number of pressure relief maneuvers reported during the interviews. Patients in the PU group could not count and record the number of pressure relief maneuvers because they were all admitted for surgical PU repair and were on bed rest as part of their treatment protocol.

Statistically significant differences were determined by univariate analysis ( $t$-test and $\chi^{2}$-test). Multivariate analysis using logistic regression was then performed for the factors for which significant between-group differences were noted to determine which factors strongly influenced the risk of PU. The Wilcoxon test was used to compare the counted number of pressure relief maneuvers with the self-reported number given during the interviews by the

\section{Table 1 Daily living-related lifestyle factors}

Wheelchair and cushion factors Number of wheelchairs and seat cushions

Length of time spent in the wheelchair

Presence or absence of transfer failure

(hitting buttocks or falling)

Protective activities

Frequency of pressure relief

Knowledge of pressure relief methods

Need for bed rest

Skin monitoring at least once a week

bathing frequency

Use of cushion in the bath and on the toilet

Urination and defecation

Technique for voiding urine and feces

Presence or absence of incontinence

Frequency of incontinence

Length of time spent voiding feces

Voiding location

Social participation
No PU group. All statistical analyses were performed using IBM SPSS version 16.0J (IBM Japan).

This study was approved by the ethics committee of Kanagawa Rehabilitation Hospital and Tokyo Metropolitan University.

\section{RESULTS}

Results are summarized in Table 2. Factors that did not significantly differ between both groups were listed in Table 2 only if they were necessary for comparison with the significantly different factors.

\section{Sociodemographic and neurological factors}

Sex, level of injury and ASIA impairment scale scores were not significantly different between the groups because these factors were matched as part of the study design. However, age, time since injury and PU history were all significantly greater in the PU group than in the No PU group (Figures 1 and 2).

\section{Risk assessment scales}

The Braden scale scores of the No PU group were slightly better than those of the PU group but this difference was not statistically significant. The PU group had significantly higher SCIPUS scores than the No PU group, indicating that the PU group had a higher risk of PUs.

\section{Lifestyle factors related to daily living}

Eight lifestyle factors were significantly different between groups. More patients in the No PU group used a wheelchair and cushion, and possessed a cushion, than in the PU group. The average number of hours in the wheelchair, and the number of baths, was also greater in the No PU group than in the PU group. More patients in the No PU group than in the PU group traveled to facilities other than their office or school at least once per month, were knowledgeable about pressure relief, and drove independently. Voiding-related factors, employee or student status, and the number of patients possessing a wheelchair were not significantly different between groups.

During the interviews, we asked all patients how many times per hour they usually performed pressure relief maneuvers. The number of patients who could answer the question was $23(74 \%)$ in the PU group and $19(63 \%)$ in the No PU group; the rest could not remember how many times they performed the actions. The hourly number of pressure relief maneuvers reported by the PU group (median, 1.0; mean \pm s.d., 2.2 \pm 3.3 ; range, $0-15$ ) did not differ significantly from the number reported by the No PU group (median, 1.5; mean \pm s.d., $1.8 \pm 1.6$; range, $0-5.5$ ). Methods of pressure relief that patients knew included lifting up, leaning to the front, side and back, and other methods as described in Table 2.

The average and median number of pressure relief maneuvers per hour as reported in the interview counted over a 1-week period, and including activities related to pressure relief in the No PU group, are shown in Figure 3. All 30 patients in the No PU group were able to complete this part of the investigation. There were significant differences between the mean number of pressure relief maneuvers reported in the interview (mean \pm s.d., $1.8 \pm 1.6$ ), the mean selfcounted number of maneuvers $(4.7 \pm 7.2)$ and the mean calculated number of maneuvers including related activities $(5.7 \pm 7.2)$. Conversely, when the medians were compared, the results of the interview (median, 1.5) and the self-counted results (2.5) did not differ significantly but the results of the interview and the median number of pressure relief maneuvers including related activities (3.5) did. We adopted the median values because of the wide distribution of results, and calculated that the patients performed pressure relief including related activities once every $17 \mathrm{~min}$. 
Table 2 Results of interviews, medical record searches and interface pressure measurements

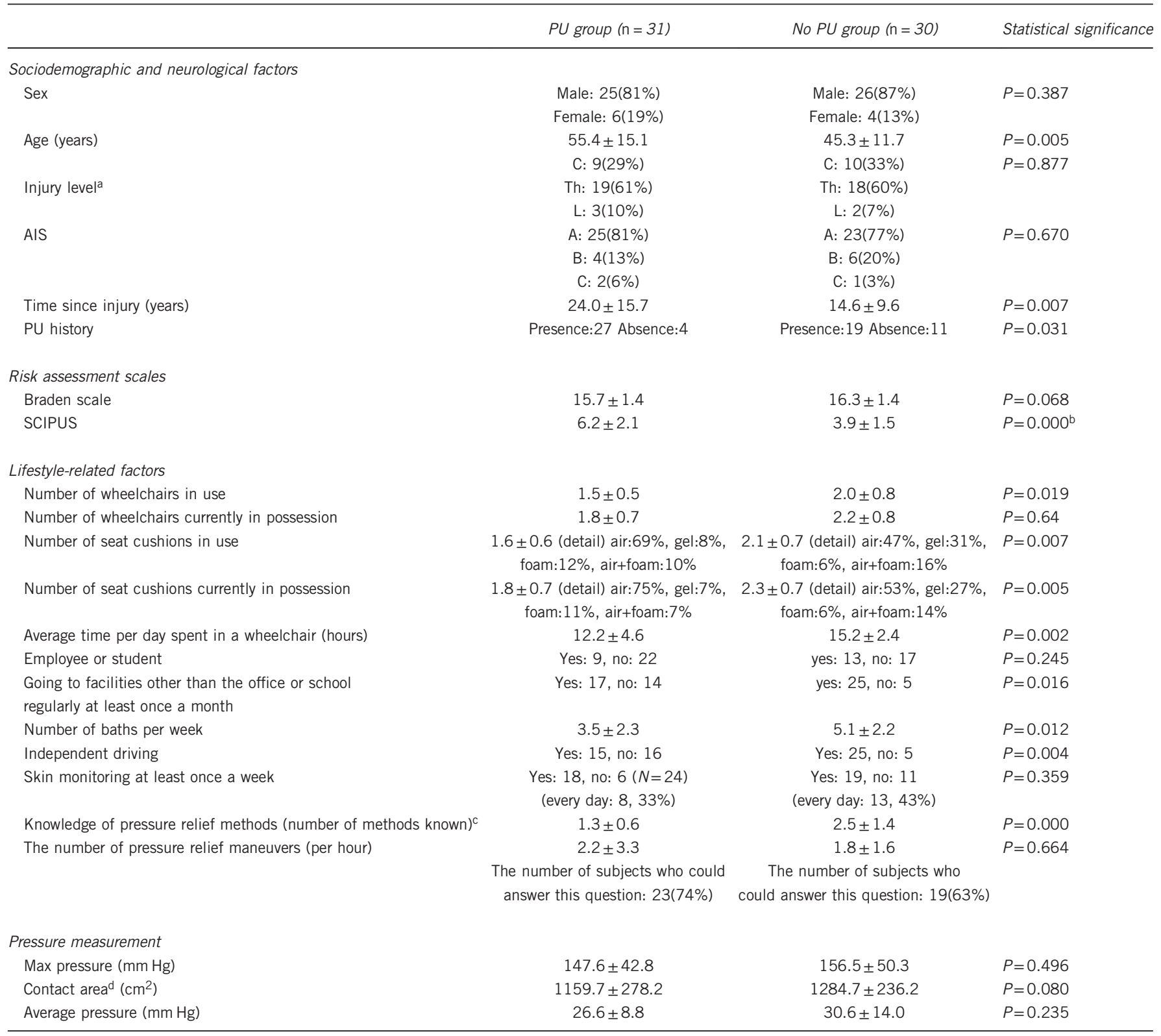

Abbreviation: AIS, ASIA impairment scale; PU, pressure ulcer; SCIPUS, spinal cord injury pressure ulcer scale.

${ }^{a}$ C, cervical, Th: thoracic, L: lumbar

bSignificantly different subscale: age $(P=0.004)$, albumin or total protein value $(P=0.000)$, hematocrit or hemoglobin value $(P=0.000)$

"We asked the subjects an open-ended question: "What kind of pressure relief methods do you know?" and they freely described to us the pressure relief methods that they were familiar with. These included lifting up, leaning their trunk (to the front, side and back), changing their posture, crossing their legs, returning to bed and using a stand up wheelchair.

dThe area with a pressure $>5 \mathrm{~mm} \mathrm{Hg}$

\section{IP measurement}

All patients in the No PU group and 24 patients in the PU group underwent IP mapping; seven $(23 \%)$ of the PU group patients were not able to undergo pressure mapping before surgical treatment of ulcers. There were no significant differences in the maximum pressure, contact area and average pressure between groups.

\section{Multivariate analysis}

Eight lifestyle factors differed significantly between groups, as did the SCIPUS scores, and these were assessed by multivariate analysis to determine which factor strongly influenced the risk of PU. Three factors had a high odds ratio for PU occurrence (Table 3), these were seat cushion possession (odds ratio $=8.110$ ), average time per day spent in a wheelchair (odds ratio $=1.581$ ) and SCIPUS score (odds ratio $=0.395)$.

\section{DISCUSSION}

Systematic review of the risk factors for PUs in patients with SCI has shown that age and injury level do not affect PU risk but sex, severity of paralysis and time since injury all strongly influence the likelihood of PU development. ${ }^{13}$ In this case-control study, injury level, sex and ASIA impairment scale did not differ significantly between groups; therefore, we were able to compare other factors that might influence PU risk. 
In contrast to the results of other studies, which indicate that age is not a risk factor for PUs, ${ }^{13}$ age differed significantly between groups. The age distribution peak was in the $60 \mathrm{~s}$ in the PU group and in the

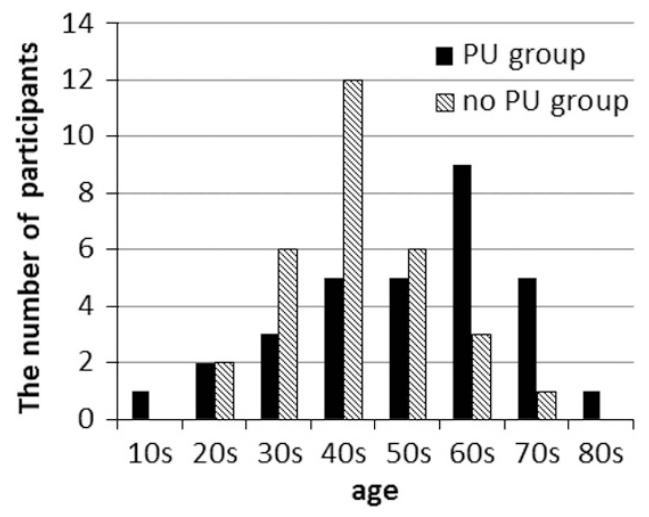

Figure 1 Age distribution of the participants.

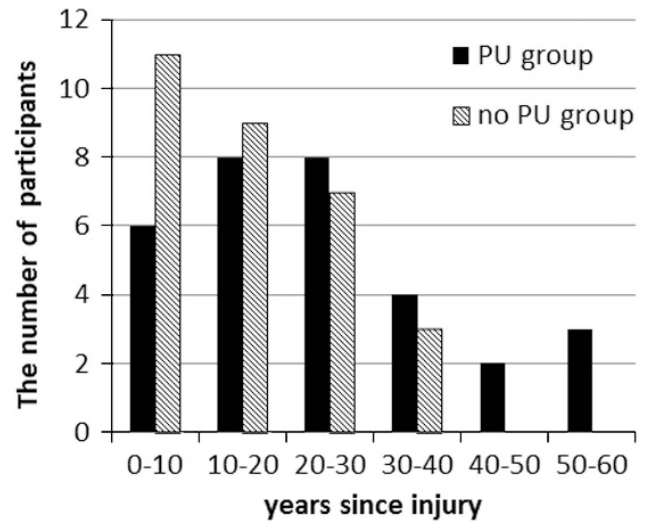

Figure 2 Distribution of the participants by number of years since injury.
$40 \mathrm{~s}$ in the No PU group; six (19\%) patients in PU group and one (3\%) patient in the No PU group were aged over 70 years (Figure 1). Patients in the PU group also had longer times since injury, with nine $(29 \%)$ having been injured $>30$ years ago compared with only three (10\%) of the patients in the No PU group (Figure 2). This suggests that the patients in the PU group were injured significantly longer ago than those in the No PU group. Therefore, we speculated that the PU group was older than no PU group because of the long time since injury.

We classified the eight lifestyle factors that differed significantly between groups into two general categories (Table 4). In general, these results suggest that No PU group patients were more active and more knowledgeable about ulcer prevention than PU group patients. Previous studies have shown that regular exercise reduces PU occurrence but simply having an active lifestyle has not been shown to have any effect. ${ }^{7,14}$ Although we did not investigate exercise in this study, we consider that an active lifestyle is a precondition to regular exercise. Therefore, patients in the No PU group might be more likely to exercise, and having an active lifestyle may be essential to PU prevention. It has previously been shown that patients with SCI benefit from the availability of aids and medical information. ${ }^{2}$ Our results suggest that the PU group patients had inadequate information about wheelchair and pressure relief activities. In addition, because the PU group was less active than the No PU group, it might have been more difficult to contact them and provide them with this information.

There were no significant between-group differences in the number of patients who performed skin monitoring at least once a week. Twothirds to three-quarters of the patients in both groups performed adequate skin monitoring. As previous studies have recommended, we also consider it necessary to educate patients with SCI about the importance of skin monitoring to prevent PUs. ${ }^{6,11}$

In the interviews, both groups reported performing a similar number of pressure relief maneuvers. However, several patients in both groups could not answer the question, which suggests that it is difficult to remember the number of pressure relief maneuvers performed because they are unconsciously practiced. Although the recommended frequency of pressure relief maneuvers is once every 15

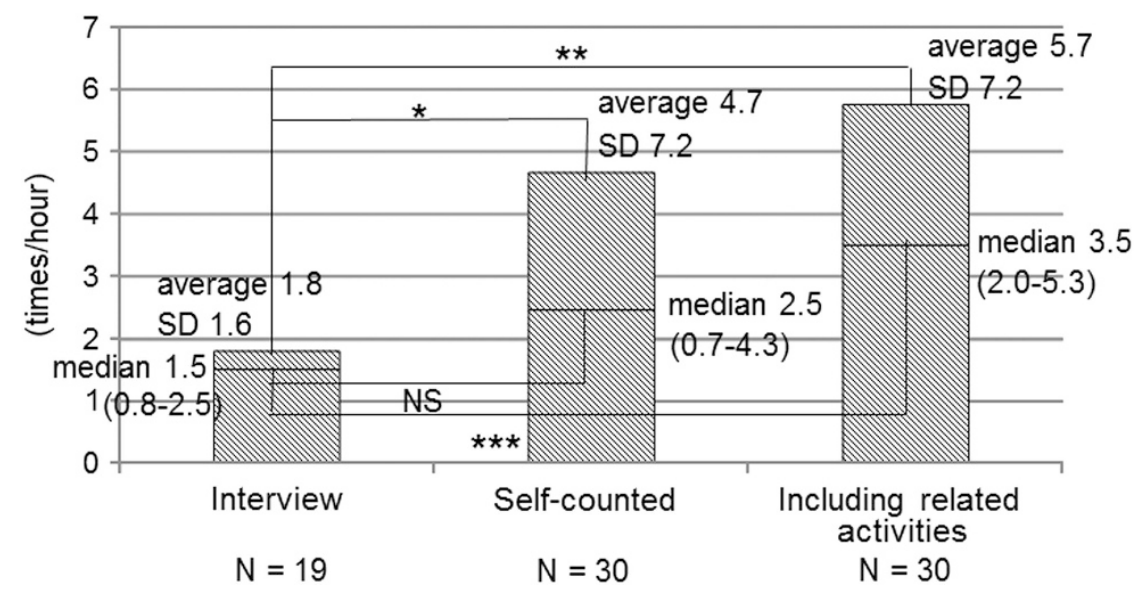

Figure 3 Comparison of the average and median number of pressure relief maneuvers as determined by interview, self-counted, and including related activities. Abbreviations NS: non-significant, s.d.: standard deviation, (25percentile-75percentile) after median, $t$-test results $* P<0.05(P=0.045)$, ${ }^{*} P<0.01(P=0.007)$, Wilcoxon test results ${ }^{* *} P<0.01 \quad(P=0.000)$. The number of subjects who could answer the question was $19(63 \%)$. When comparing means, the self-counted number of pressure relief maneuvers and self-counted number including related activities were significantly larger than the number of maneuvers reported in the interview. When comparing medians, the results of the interview and the median number of pressure relief maneuvers including related activities significantly differed. Based on calculations using the median values, No PU participants performed pressure relief including related activities once every $17 \mathrm{~min}$. 
Table 3 Multivariate analysis of lifestyle factors and risk assessment scale scores strongly associated with pressure ulcer development

\begin{tabular}{|c|c|c|c|c|}
\hline & $B$ & $P$ & $O R$ & $\mathrm{Cl}$ \\
\hline Average time per day spent in a wheelchair & 0.458 & 0.004 & 1.581 & $1.154-2.166$ \\
\hline
\end{tabular}

Abbreviations: $\mathrm{Cl}$, 95\% confidence interval; OR, odds ratio; $P, P$-value; $\beta$, partial regression coefficient. $83.3 \%$ of variance explained by model. Result of Hosmer-Lemeshow test was $P=0.089$.

Table 4 Classification of eight factors that significantly differed between groups

\begin{tabular}{ll}
\hline Category & Factors \\
\hline Active lifestyle & $\begin{array}{l}\text { Average hours spent in a wheelchair } \\
\text { Going to facilities at least once a } \\
\text { month (except for the office or school) } \\
\text { Number of baths per week } \\
\text { Driving themselves }\end{array}$ \\
Information about wheelchair use & $\begin{array}{l}\text { Number of wheelchairs in use } \\
\text { and pressure relief techniques }\end{array}$ \\
& $\begin{array}{l}\text { Number of cushions in use } \\
\text { Knowledge of pressure relief } \\
\text { techniques }\end{array}$ \\
\hline
\end{tabular}

min, ${ }^{10}$ previous studies have shown that community-dwelling patients with SCI do not engage frequently enough in pressure relief activities. ${ }^{11}$ However, in our study, the patients in the No PU group practiced pressure relief activities at almost the recommended frequency. We consider this result useful for planning PU prevention programs in the future.

In this study, contrary to our expectations, the results of IP measurements did not differ significantly between groups. It has been reported that it is difficult to identify an IP damage threshold because IP cannot measure internal pressure. ${ }^{15}$ Our results also suggest that identification of an IP damage threshold is difficult. However, in contrast, studies comparing cushions and postures have reported differences in IP. ${ }^{16,17}$ As the results of these studies suggest, comparison of IP is helpful in the clinical setting when we must choose the appropriate cushion for the patient. We consider relative comparisons of IP to be more useful than selecting an absolute damage threshold.

Logistic regression revealed that the number of seat cushions possessed, average time per day spent in a wheelchair and the SCIPUS score were highly associated with PU development. Our findings regarding the number of cushions possessed suggest that the patients in the No PU group were concerned about their cushions and updated them regularly. The mean time since injury was over 20 years in the PU group and over 10 years in the No PU group; this result suggests that it is important for SCI patients to be aware of, and to update, their cushions. Cushions may deteriorate when used for such a long period, and their ability to redistribute pressure may decrease with age.

SCIPUS has been reported to be effective for PU prediction, ${ }^{18}$ and is recommended in the Japanese guidelines for the prevention of ulcers. ${ }^{19}$ We speculate that SCIPUS scores differed significantly between groups in this study because SCIPUS includes age and blood test data that the Braden scale does not include.

\section{Limitations}

The patients in this study were from a single community, Kanagawa, Japan. Thus, our results may be specific to people in this area. In addition, cases were hospitalized and controls were community dwelling; this difference in setting may have affected the recall of lifestyle factors. The connection with risk factors not examined in this study, including substance abuse (alcohol, drugs) and psychological factors, ${ }^{13}$ is unidentified. This study involved the comparison of many factors between groups, which could lead to alpha inflation or type 1 errors.

\section{CONCLUSIONS}

This study showed that eight factors associated with daily living differed significantly between the PU and No PU groups. Factors that were strongly associated with PU occurrence were the number of seat cushions possessed, average time per day spent in a wheelchair and SCIPUS scores. These results suggested that the No PU group patients adopted an active lifestyle and had much more information about wheelchair, cushion and pressure relief techniques. In contrast to other studies, age also differed significantly between groups because of the long time since injury. IP measurements did not differ significantly between groups. In the No PU group, pressure relief maneuvers, including related activities, were performed once every $17 \mathrm{~min}$. Our findings may be useful for developing a pressure ulcer prevention program for community-based patients with SCI.

\section{DATA ARCHIVING}

There were no data to deposit.

\section{CONFLICT OF INTEREST}

The authors declare no conflict of interest.

1 Biglari B, Büchler A, Reitzel T, Swing T, Gerner HJ, Ferbert T et al. A retrospective study on flap complications after pressure ulcer surgery in spinal cord-injured patients. Spinal Cord 2014; 52: 80-83.

2 van Loo MA, Post MWM, Bloemen JHA, van Asbeck FWA. Care needs of persons with long-term spinal cord injury living at home in the Netherlands. Spinal Cord 2010; 48: 423-428.

3 Mathew A, Samuelkamaleshkumar S, Radhika S, Elango A. Engagement in occupational activities and pressure ulcer development in rehabilitated South Indian persons with spinal cord injury. Spinal Cord 2013; 51: 150-155.

4 Garber SL, Rintala DH. Pressure ulcers in veterans with spinal cord injury: a retrospective study. J Rehabil Res Dev 2003, 40: 433-441.

5 Royal College of Nursing. Clinical Practice Guidelines: Pressure Ulcer Risk Assessment And Prevention: Recommendations 2001. RCN Publications: London, UK, 2007; 18

6 European Pressure Ulcer Advisory Panel and National Pressure Ulcer Advisory Panel. Treatment of Pressure Ulcers: Quick Reference Guide. National Pressure Ulcer Advisory Panel: Washington, DC, USA, 2009; 19-21.

7 Krause JS, Broderick L. Patterns of recurrent pressure ulcers after spinal cord injury: identification of risk and protective factors 5 or more years after onset. Arch Phys Med Rehabil 2004; 85: 1257-1264.

8 Guihan M, Hastings J, Garber SL. Therapists' roles in pressure ulcer management in persons with spinal cord injury. J Spinal Cord Med 2009; 32: 560-567.

9 Crawford SA, Strain B, Gregg B, Walsh DM, Porter-Armstrong AP. An investigation of the impact of the Force Sensing Array pressure mapping system on the clinical judgement of occupational therapists. Clin Rehabil 2005; 19: 224-231.

10 Consortium for Spinal Cord Medicine Clinical Practice Guideline. Pressure Ulcers: What You Should Know- A Guide for People with Spinal Cord Injury. Paralyzed Veterans of America: Washington, DC, USA, 2002; 15-18.

11 Yang YS, Chang GL, Hsu MJ, Chang JJ. Remote monitoring of sitting behaviours for community-dwelling manual wheelchair users with spinal cord injury. Spinal Cord 2009; 47: 67-71. 
12 Mortenson WB, Miller WC, the SCIRE Research Team. A review of scales for assessing the risk of developing a pressure ulcer in individuals with SCI. Spinal Cord 2008; 46: 168-175.

13 Gélis A, Dupeyron A, Legros P, Benaïm C, Pelissier J, Fattal C. Pressure ulcer risk factors in persons with spinal cord injury Part 2: the chronic stage. Spinal Cord 2009; 47: 651-661.

14 Sumiya T, Kawamura K, Tokuhiro A, Takechi H, Ogata H. A survey of wheelchair use by paraplegic individuals in Japan. Part 2: prevalence of pressure sores. Spinal Cord 1997; 35: 595-598.

15 Oomens CWJ, Loerakker S, Bader DL. The importance of internal strain as opposed to interface pressure in the prevention of pressure related deep tissue injury. J Tissue Viability 2010; 19: 35-42.

\section{APPENDIX}

SCIPUS (spinal cord injury pressure ulcer scale)

1) Level of activity

2) Mobility

3) Complete $\mathrm{SCl}$

4) Urine incontinence or constant moistness

5) Autonomic dysreflexia or severe spasticity

6) Age (years)

7) Tobacco use/smoking
16 Brienza DM, Karg PE, Geyer MJ, Kelsey S, Trefler E. The relationship between pressure ulcer incidence and buttock-seat cushion interface pressure in at-risk elderly wheelchair users. Arch Phys Med Rehabil 2001; 82: 529-533.

17 Urasaki M, Nakagami G, Sanada H, Kitagawa A, Tadaka E, Sugama J. Interface pressure distribution of elderly Japanese people in the sitting position. Disabil Rehabil Assist Technol 2011; 6: 38-46.

18 Guihan M, Garber SL, Bombardier CH, Goldstein B, Holmes SA, Cao L. Predictors of pressure ulcer recurrence in veterans with spinal cord injury. J Spinal Cord Med 2008; 31: 551-559.

19 The Japanese Society of Pressure Ulcers Guideline Revision Committee: JSPU guidelines for the prevention and management of pressure ulcers. 3rd edn. Jpn J PU 2014; 16: $12-90$.
( ) ambulatory

1 ( ) wheelchair

4( ) bed

8) Pulmonary disease

9) Cardiac disease

10) Blood glucose levels: $>110 \mathrm{mg} \mathrm{dl}^{-1}$

11) Renal disease

1( ) limited

3( ) immobile

$\mathrm{O}$ ( ) no

1( ) yes

0 ( ) no

1( ) yes

$\mathrm{O}$ ( ) no

$1($ ) yes

0()$\leqslant 34$

1 ( ) 35-64

2()$\geqslant 65$

$O($ ) never

1( ) former
3( ) current

$\mathrm{O}$ ( ) no

2( ) yes

$\mathrm{O}$ ( ) no

1( ) yes

$\mathrm{O}$ ( ) no

1( ) yes

O( ) no

1( ) yes

$\mathrm{O}$ ( ) no

1( ) yes

$\mathrm{O}$ ( ) no

1( ) yes

$\mathrm{O}$ ( ) no

1( ) yes

$\mathrm{O}$ ( ) no

1( ) yes
Risk: low 0-2, moderate 3-5, high 6-8, very high 9-25 